\title{
- Cephalo-anatomy of Meitei Males of Manipur, India: A Diachronic Study
}

IJCRR
Section: Healthcare
ISI Impact Factor
$(2019-20): 1.628$
IC Value (2019): 90.81
SJIF (2020) = 7.893
CC) (1) @

\section{Thoudam Bedita Devi ${ }^{1}$, Soibam Jibonkumar Singh ${ }^{2}$}

'Assistant Professor, Department of Anatomy, Sikkim Manipal Institute of Medical Sciences, Upper Tadong, Gangtok, Sikkim- 737102, India; ${ }^{2}$ Professor, Department of Anthropology, Manipur University, Canchipur, Imphal, Manipur-795003, India.

\section{ABSTRACT}

Introduction: Each individual on the surface of this earth has unique anatomical features which distinguishes him from others. Among such unique features, cephalo-facial characters are the most commonly used parameters employed for personal identification. Though cephalo-facial anatomy of one ethnic group differs from another, yet within the same ethnic group it may experience diachronic change. Few works have been carried out specifically on cephalo-facial features of some selected population groups of India. But no such exhaustive study has been carried out on the Meiteis of Manipur valley from a diachronic approach.

Aims and Objectives: The present work aims at profiling a database of the cephalic anatomy of the Meitei adult male population of Manipur on one hand and diachronic comparison of the present findings with the findings of Singh (1992) who carried out a similar work almost three decades back from now with a view to examine if there is any secular micro-evolutionary change taking place in cephalic on the other.

Methodology: The present study is conducted on randomly selected 1600 Meitei adult males of Manipur valley whose age range from 21 to 60 years. Two direct cephalo-facial measurements i.e. maximum head length and maximum head breadth have been measured employing Weiner and Lourie (1969) technique and corresponding cephalic index was calculated and classified according to the conventional categories of Labzelter and Saller (1968).

Results: Based on the findings of the measures of cephalic dimensions, Meiteis dominantly have medium to long head length and head breadth with an overall head shape ranging between mesocephalic to brachycephalic. The findings reveal that among the adult Meitei males, both maximum head length and maximum head breadth have increased from the last generation to the present generation. While the cephalic index index of the present generation have lower mean value than the previous generation showing statistically significant difference.

Conclusion: The present findings therefore reveal a positive secular change towards increasing head length and head breadth while a negative secular trend in cephalic index. The database derived from the findings of the present study shall definitely have clinical applications in the field of cranio-facial surgery as well as in forensic application particularly in personal identification and of cranial reconstruction of the victims of the said ethnic community.

Key Words: Manipur, Meitei, Cephalo-facial, Cephalic Index, Brachycephalic, Dolichocephalic

\section{INTRODUCTION}

One of the most important objectives of anatomy and anthropology lies in understanding the morphological and anatomical variation from an evolutionary and racial point of view of humankind both at the individual as well as population-level. Study of morphological and anatomical features of man, a concern of the anthropologist and anatomist have played a significant role. As far as the biological understanding of personal, ethnic and racial identity is concerned, the cephalo- facial part is the most important dissected component of the human body.

Cephalo-facial anthropology is indeed an important tool for determining the morphological and anatomical features of the head and face, which can steer the anthropological, anatomical and forensic investigations. ${ }^{1}$ At the same time, cephalo-facial dimensions and indices are the most straight forward and most efficient tool for studying racial differences ${ }^{2}$ population variations and sexual dimorphism ${ }^{3}$ identification

\section{Corresponding Author:}

Soibam Jibonkumar Singh, Professor, Department of Anthropology, Manipur University, Canchipur, Imphal, Manipur-795003, India. E-mail: drjibonkumar@yahoo.com

ISSN: 2231-2196 (Print)

Received: 01.01 .2021
ISSN: 0975-5241 (Online)

Revised: 22.02 .2021
Accepted: 02.04 .2021
Published: 10.08 .2021 
of race, ethnicity, gender, age. ${ }^{4}$ Cephalo-facial dimensions experience change during growth and development period and reach its peak growth between 16-20 years of life and finally attains maturity after that.., 6 Variation in head and face depend on many factors such as ethnicity, genetic influence, traditions, nutrition, certain pathological conditions, environment and climate. ${ }^{7}$

Hence driven by the need necessitates studies on the secular trend in the cephalic dimensions and indices and Meitei male populations. Secular trend in physical growth pattern is one of the biological evolutionary phenomena taking place almost in all human population groups of the world. Study on secular trend for the first time was described on human stature by Quetelet in $1835 .{ }^{8}$ But now it is not restricted to the only stature but also to axial, appendicular, facial parts of the body as well.

Though quite many scholars have worked on the secular trend in cephalo-facial measurements on different population groups of the world, the report from the Indian population is comparatively very few. With only a few such studies in North East India, little is known concerning the cephalofacial dimension conducted on the population of Manipur. Such a scanty nature of data on Indian population more particularly among the populations of North East India and more specifically of the population of Manipur calls for undertaking a pioneering research. Steered by this fact and not having done any work inter-generational variation of cephalic anatomy, the present research work has been carried out on the Meitei male population of Manipur valley with a view to document their cephalic profile on one hand and examine if any secular trend has taken place in cephalic anatomy from earlier generation to the present generation on the other. Keeping this in view, the present study is undertaken with an objective of preparing a database of cranial profile of the studied population with a view to provide a baseline data for anatomical and clinical application in the field of cranial surgery and reconstruction as well as forensic application of personal identification.

\section{MATERIALS AND METHODS}

The present study on the cephalo-facial profile has been carried exclusively on 1600 Meitei adult males who belonged to the age range of 21-60 years representing 400 each from four valley districts of Manipur i.e Imphal West, Imphal East, Bishnupur and Thoubal district selected randomly through multi stage random sampling method. This cross sectional sample comprising of 400 individuals each represents the four different age groups (21-30, 31-40, 41-50, 51-60) years of age. Only those individuals with no cephalo-facial surgery/anomalies and who volunteered and gave ethical clearance and written consent were selected as subjects of the present study. The following two cephalometric measurements were taken with the help of Martin's sliding caliper and blunted knob Martin's spreading caliper following the techniques laid down by Weiner and Lourie (1969) on the subjects sited on a chair in a relaxed condition with the head oriented in eye ear plane. ${ }^{9}$

\begin{tabular}{|c|c|c|}
\hline Measurement & Landmark & $\begin{array}{l}\text { Instrument } \\
\text { used }\end{array}$ \\
\hline $\begin{array}{l}\text { 1. Maximum } \\
\text { Head Length } \\
\text { (MHL)- straight } \\
\text { distance between } \\
\text { Glabella and } \\
\text { Opisthocranion }\end{array}$ & $\begin{array}{l}\text { Glabella (g)- promi- } \\
\text { nent point in be- } \\
\text { tween two eyebrows } \\
\text { just above the nasal } \\
\text { root in mid sagittal } \\
\text { line } \\
\text { Opisthocranion(op) } \\
\text { - most posterior } \\
\text { point on the head in } \\
\text { mid sagittal plane }\end{array}$ & $\begin{array}{l}\text { Spreading cali- } \\
\text { per with blunt } \\
\text { knob }\end{array}$ \\
\hline $\begin{array}{l}\text { 2. Maximum Head } \\
\text { Breadth (MHB)- } \\
\text { straight distance } \\
\text { between Euryon } \\
\text { to Euryon }\end{array}$ & $\begin{array}{l}\text { Euryon (eu) - most } \\
\text { lateral point on the } \\
\text { lateral prominence } \\
\text { of parietal bone }\end{array}$ & $\begin{array}{l}\text { Spreading cali- } \\
\text { per with blunt } \\
\text { knob }\end{array}$ \\
\hline
\end{tabular}

Based on the two measurements, the following four relevant indices were calculated.

Cephalic Index $: \frac{\text { Maximum Head Breadth }}{\text { Maximum Head Length }} \times 100$

The two measurements and the cephalic index were classified according to the conventional categories of Labzelter and Saller (1968). ${ }^{10}$

The statistical constants such as Mean, Standard Deviation, and their respective standard error were calculated. Independent sample t-test has been used to compare the mean values with previous works. To determine statistical significance of qualitative data, Chi-Square test has also been applied. All the statistical calculations were calculated using SPSS version 22.

\section{RESULTS}

Steered by the hypothesis that changes might have not occurred in cephalo facial measurements between the present Meitei Population and past Meitei population of Manipur, the following section delve into detail comparison of the cephalo facial parameters of present-day adult male Meitei population of Manipur valley with that of the findings of the Singh who carried out similar work on the same population three decades back from now in a diachronic approach with an aim to see if any changes have occurred in these characters, thereby leading and directing to a secular change be it a 
positive one or negative one. A comparative account of these two studies is presented in the following section in a systematic and scientific approach.

As evident from table 1 that the frequency per cent distribution of maximum head length of the past and present Meitei male population fall within the classification ranging from very short to very long among the past population and short to very long among the present population. On qualitative comparison of the maximum head length of the past and present population, it is evident that highest frequency percent of maximum head length is observed in the medium class $(43 \%)$, followed by short $(31 \%)$, long $(20 \%)$, very short (4\%) and very long (2\%) being the least for the past population. However, among the Meiteis of present population the highest is observed in long category ( $40 \%)$ followed by medium $(38 \%)$, very long $(13.50 \%)$ and short $(8.50 \%)$. When statistically compared using chi-square test, a significant difference $\left(\chi^{2}=31.651\right.$, table 2$)$ is observed between the past and present male Meitei population of Manipur valley.

On quantitative comparison, the present population shows higher mean values $(18.65 \pm 0.03 \mathrm{~cm})$ than the past population $(18.05 \pm 0.06 \mathrm{~cm})$ thereby showing a statistically significant difference $(t=8.49$, table 3$)$. These findings, therefore reveals that a positive secular trend in the dimension of maximum head length is taking place from past generation to the present generation.

As regards the frequency per cent distribution of maximum head breadth, the highest frequency is observed in the medium-class which range between 148.0-155.0 $\mathrm{mm}$ in both the present $(49.75 \%)$ and past $(49 \%)$ populations. Medium class of maximum head breadth is followed by narrow (35\%) among the past population while broad (27.00\%). A very sharp variation is observed in the distribution of narrow category of head breadth between the past (35\%) and present $(18.25 \%)$ population. Analysis of the qualitative comparison also revealed a statistically significant difference $\left(\chi^{2}=8.618\right.$, table 2$)$. Comparison of the mean values of the past population $(\overline{\mathrm{x}}=14.99 \pm 0.05 \mathrm{~cm})$ and present population $(\overline{\mathrm{x}}=15.27 \pm 0.03 \mathrm{~cm})$ also shows a positive secular trend as revealed by the observed statistically significant difference $(\mathrm{t}=4.80$, table 3$)$.

Considering the comparison of the head shape expressed as cephalic index (table 1), brachycephalic head is predominantly the highest frequently occurring class observed in both the population (Past study $41.00 \%$ and Present study, $39.00 \%$ ). At the same time both the population displays a similar preponderance order of Brachycephalic $>$ Mesocephalic $>$ Hyperbrachycephalic $>$ Dolichocephalic $>$ U1trabrachycephalic and Hyperdolichocephalic. The similarity in the frequency per cent distribution and preponderance order as well confirms statistically non-significant difference $\left(\chi^{2}=3.416\right.$, table 2$)$.
However, when quantitatively compared, the mean value of the present population is experiencing a decreasing trend from the past. The difference of 1.36 in between the mean value of the past $(\overline{\mathrm{x}}=83.25 \pm 0.43)$ and present $(\overline{\mathrm{x}}=81.89 \pm$ 0.22 ) population reflects a statistically significant difference $(\mathrm{t}=2.84$, table 3$)$.

\section{DISCUSSION}

Based on the objectives of the work, the data collected from 1600 (400 each from a districts) Meitei male adults within the age range 21-60 years of age have been statistically treated and the findings presented under the caption results. This section shall exclusively deal with the analytical discussion of the findings of the aforesaid results.

Many of the earlier researchers studied racial affinity of the Meiteis and described as Mongoloid owing to the presence of mongoloid features. ${ }^{11,12,13}$ The present findings too reveal that Meitei males of Manipur valley possessing mongoloid features with respect to cephalic characters in general by having medium to long head length and head breadth with an overall head shape ranging between mesocephalic to brachycephalic. All these observed anatomical features of Meitei Males are mongoloid features and is in agreement with the findings of other scholars who worked on different mongoloid populations. ${ }^{14,15,16,17}$

It has been observed that Maximum Head Length and Maximum Head Breadth of the Meitei population have experienced a positive secular change towards increasing size from the last generation to the present generation. In short Meiteis of the present generation have longer and broader head than the previous generation, Looking into the cephalic index, the Meitei population of the present generation have lower mean value $(\bar{X}=81.89 \pm 0.22)$ than the previous generation $(\bar{X}=83.25 \pm 0.43)$ showing a negative secular trend showing statistically significant difference $(\mathrm{t}=2.84$,). Similar findings showing a negative secular trend was also reported by Golalipour et al. who studied the Japanese population. ${ }^{18}$ This negative trend is because of the more significant increase in head length $(0.60 \mathrm{~mm})$ as compared to head breadth $(0.28 \mathrm{~mm})$. This finding of the present study is in agreement with those reported by Sanna et al. ${ }^{19}$ who studied the Sardinian, but contrasting to that of among whom there is a more significant increase in head breadth as compared to head length and among Japanese children. ${ }^{20,21}$ Therefore, as a result of decreasing cephalic index, the population is gradually experiencing debrachycephalization. Similar findings were also reported among the Sardinian Children ${ }^{22,23}$; among the Hungarian $^{24,25}$; among the Zena children ${ }^{26}$; among the Croa$\operatorname{tian}^{27}$ among Russian children ${ }^{28}$ and among Ogbia tribe of Bayelsa State. ${ }^{29}$ However, the mean values of the cephalic index of both the Meitei populations indicate that their head 
shape falls under the brachycephalic category. Though the mean value shows a significant difference, the frequency per cent distribution of different head form between the past and present population still remains more or less the same thereby revealing no statistically significant difference between the populations as evidenced from chi-square value $\left(\chi^{2}\right.$ $=3.416)$. Even though the size of the head, i.e. head length and head breadth increases, head shape remains more or less unchanged in both the populations because of the fact that head shape or form depends more on pre-established constitutional hereditary factors and less on environmental factors as compared to head size for which environmental factors have a great influence on genetic fact.

\section{CONCLUSION}

On the basis of the results obtained, it can be concluded that the cephalic features i.e. maximum head length and maximum head breadth has experienced an increase in dimensions resulting to a diachronic positive secular change during the last generation. On the other hand the ratio between head length and head breadth i.e. cephalic index of the present Meitei males has experienced a decreasing value from the last generation thereby revealing a negative secular change even though the conventional category of head shape remains the same.

\section{ACKNOWLEDGEMENT}

We are grateful to the Department of Anthropology, Manipur University, Canchipur for rendering us to utilize the instruments during the course of this study/work and also our heartfelt thanks to all the participants for their sincere co-operation during the work.

\section{Conflict of Interest : Nil}

\section{Source of funding: NIL}

Authors contribution : Data collection and analysis of the same was carried out by the first author and over all supervision and guidance during the course of work was done by the second author.

\section{REFERENCES}

1. Cvetkovic, M., Stevo, N. and Milos, N. Secular change in cephalic index- a study of Serbian School Children, GENETIKA 2014; 46 (2), 561-568.

2. Chaturvedi, R. P, Harneja, N. K. A Cephalometric study of Human Skulls. J. Anat. Soc. India, 1963; 12, 93-96.

3. Ilayperuma, I. Evaluation of cephalic Indices: A clue for racial and Sexual diversity. Int. J. Morphol,2011; 29 (1), 112-117.

4. Sol, D. M. Cephalic Index in a group of Mapuche individuals in the IX Region of Chile.Int. J. Morphol, 2005;23 (3), 241-246.
5. Kondu, S., Wakatsuki, E., Shibagaki, H. A. Somatometric study of the head and face in Japanese adolescents. Okajimas Folia Anat Japan, 1999; 76 (4), 179-85.

6. Knutson, B., Momenan, R., Rawlings, R. R., Fong, G. W. and Hommer, D. Negative association of neuroticism with brain volume ratio in healthy humans. Biol. Psychiatry,2001; 50,685-690.

7. Rexhepi, A., Meka, V. Cephalo-facial morphological characteristics of Albanian Kosova population. Int. J. Morphol, 2008; 26 (4), 935-940.

8. Smith, B. H., Garn, S. M., Hunter, W. S. Secular trend in face size. Angle Orthod, 1986; 56, 196-204.

9. Weiner, J. S, Lourie J. A. Human Biology, A Guide to Feild Methods. IBP Handbook no.9. Blackwell Scientific Publications, Oxford;1969.

10. Labzeltar, Saller, A.. Reproduced from Singh, I. P, Bhasin, M. K. Anthropometry. New Delhi. Bhart Bhavan. Kem Publishers; 1968.

11. Sanajaoba, N. Manipur Past and Present. Mital Publication, New Delhi. $1 ; 1988$

12. Singh, C. B. The Ethnonym, Meitei. In Manipur Past and Present. $1 ; 2003 ; 72-81$.

13. Kabui, G. History of Manipur. National Publishing House, New Delhi;1989.

14. Xu, B, Wang, Y, Ma, J, Li, M, Xu. L. A computer aid study on the craniofacial features of Archang race in Yunnan province of China. Hua Xi Kou Qiang Yi Xue Za Zhi, 2011; 19 (6), 394-396.

15. Kroeber, A. L. American Culture and the Northwest Coast, American Anthropologist, 1923; 25, 1 -25.

16. Kasika, R. L. C, Brozon, T. Comparative study of craniofacial morphology in Japanese and Australian aboriginal population. Hum Biol, 1993; 65(5),821-834.

17. Zheng, L. B. Physical characteristics of Chinese Hakka. Sci China Life Sci, 2013;56, 541-551.

18. Golalipour, M. J, Jahanshahi. M., Haidari. K. Morphological evaluation of head in Turkman males in Gorgan- North of Iran. Int. J. Morphol, 2007; 25 (1), 99-102.

19. Sanna, E, Palmas I. Change in Body and Head Dimensions in urban Sadinian children (3-5years) from 1986-2001. Ann. Hum. Biol; 2008;12: 782-791.

20. Kouchi, M, Vojdani, Z. Brachycephalization in Japan has ceased. Am J Phys Anthropol, 2000;112, 339-347.

21. Heravi, F, Zieace, H. Assessing the importance of Cephalic and Facial indices in a group of 12 years old boys in Mashhad. Behesti University. Dent. J; 2002; 20, 119-124.

22. Sanna, E, Soro, M. R. Anthropometric changes in urban Sardinian children 7-10 years between 1975-1976 and 1996.Ann. Hum. Biol; 2000; 12: 782-791

23. Sanna. E, Palmas I. Change in Body and Head Dimensions in urban Sadinian children (3-5years) from 1986-2001. Ann. Hum. Biol.; 2008;12: 782-791.

24. Gyenis G. Rapid change in head and facial measurements in university students in Hungary. Anthropol. Anz; 1994; 52, 149-158.

25. Magyar. L, Bellovits. O, Bujdoso, G. Changes in anthropological data of Hungarian child and adult population during the last thirty years based on family study conducted by the Department of Forensic Medicine at Budapest. Anthropol. Anz.;2006; 64: 227-241.

26. Zellner. K, Jaeger, U, Kromeyer. H. K. The phenomenon of debrachycephalization in Jena School Children. Anthropol. Anz.; 1998; 56, 301-321.

27. Buretic. A, Tomljanovic. A. A, Giacomett. J, Ostojic. A. S, Kapovic. A. M. Sex-specific differences of craniofacial traits in Croatia: The impact of environment in a small geographic area. Ann. Hum. Biol; 2007; 34, 296-314. 
28. Godina. E. Z. Secular trend in some Russian populations. Anthropol. Anz; 2011;68, 367-377.
29. Eroje. M. A., Fawehimmi. H. R, Jaja. B. N, Yasker. I. Cephalic Index of Ogbia Tribe of Bayesla State. Int. J. Morphol; 2010; 28 (2), 389-392.

Table 1: Frequency Percent Distribution of Maximum Head Length of Manipur Meitei Male Population of Past and Present According to Conventional Categories

\begin{tabular}{|c|c|c|c|c|c|}
\hline \multirow[t]{2}{*}{ Classification } & \multirow{2}{*}{ Range } & \multicolumn{2}{|c|}{ Singh (1992) } & \multicolumn{2}{|c|}{ Present Study } \\
\hline & & f & $\%$ & f & $\%$ \\
\hline \multicolumn{6}{|c|}{ Maximum Head Length (in mm) } \\
\hline Very short & $\infty-169.0$ & 4 & 4 & o & o \\
\hline Short & $170.0-177.0$ & 31 & 31 & 136 & 8.50 \\
\hline Medium & $178.0-185.0$ & 43 & 43 & 608 & 38.00 \\
\hline Long & $186.0-193.0$ & 20 & 20 & 640 & 40.00 \\
\hline Very long & $194.0-\infty$ & 2 & 2 & 216 & 13.50 \\
\hline Total & & 100 & 100 & 1600 & 100 \\
\hline \multicolumn{6}{|c|}{ Maximum Head Breadth (in mm) } \\
\hline Very Narrow & $\infty-139.0$ & o & o & 28 & 1.75 \\
\hline Narrow & $140.0-147.0$ & 35 & 35 & 292 & 18.25 \\
\hline Medium & $148.0-155.0$ & 49 & 49 & 796 & $49 \cdot 75$ \\
\hline Broad & $156.0-163.0$ & 16 & 16 & 432 & 27.00 \\
\hline Total & & 100 & 100 & 1600 & 100 \\
\hline \multicolumn{6}{|l|}{ Cephalic Index } \\
\hline Hyperdolichocephalic & $\infty=70.9$ & 1 & 1 & 12 & 0.75 \\
\hline Dolichocephalic & $71.0-75.9$ & 3 & 3.00 & 80 & 5.00 \\
\hline Mesocephalic & $76.0-80.9$ & 29 & 29.00 & 588 & 36.75 \\
\hline Brachycephalic & $81.0-85.4$ & 41 & 41.00 & 624 & 39.00 \\
\hline Hyperbrachycephalic & $85.5-90.9$ & 25 & 25.00 & 264 & 16.50 \\
\hline Ultrabrachycephalic & $91.0-\infty$ & 3 & 3.00 & 32 & 2.00 \\
\hline Total & & 100 & 100 & 1600 & 100 \\
\hline
\end{tabular}

Table 2: $\chi^{2}$ value for comparison Cephalic Measurements of Past and Present Meitei Male Population of Manipur Valley

\begin{tabular}{lccc} 
Parameter & \multicolumn{1}{c}{} & $\mathrm{d}$ f & P-value \\
Maximum Head Length (MHL) & $31.651^{*}$ & 3 & 0.00 \\
Maximum Head Breadth (MHB) & $8.618^{*}$ & 2 & 0.01 \\
Cephalic Index (CI) & 3.416 & 3 & 0.33 \\
\hline
\end{tabular}

Table 3: Obtained Statistical Constants for Cephalic Measurements of Past and Present Meitei Male Population of Manipur Valley

\begin{tabular}{llcccccc} 
Parameter & Population & Range & Mean \pm SE & Std Dev. \pm SE & Difference of Mean & t-value & P value \\
MHL & Singh 1992 & $16.8-19.5$ & $18.05 \pm 0.06$ & $0.63 \pm 0.04$ & & & \\
& Present Study & $17.1-21.1$ & $18.65 \pm 0.03$ & $0.66 \pm 0.02$ & 0.6 & $8.94^{*}$ & 0.001 \\
(in cm) & & & & & & \\
MHB & Singh 1992 & $14.0-16.2$ & $14.99 \pm 0.05$ & $0.50 \pm 0.04$ & & & \\
& Present Study & $13.2-17.2$ & $15.27 \pm 0.03$ & $0.60 \pm 0.02$ & & & \\
(in cm) & & & & & & & \\
CI & Singh 1992 & $71.81-92.30$ & $83.25 \pm 0.43$ & $4.31 \pm 0.31$ & & & \\
& Present Study & $59.68-95.45$ & $81.89 \pm 0.22$ & $4.32 \pm 0.15$ & & $2.84^{*}$ & 0.01 \\
\hline
\end{tabular}

